Reilly, Rosemary C.

Encyclopedia of case study research

\title{
Participatory case study
}

The participatory case study is a mode of case study research that involves the participants, local groups, or the community in all phases of the research process, from conceptualizing the study to writing up and disseminating the findings. It is ideologically oriented in its focus and explicitly emancipatory in its goals. It generally takes the position of social critic, and proposes radical change in social structures/processes, as well as reformulating the entire approach to research, voice, power, and knowledge production/use. Emerging from the liberation and anti-colonial philosophical approach of Paulo Freire, participants are not incidental to the curiosity of the researcher, but are experts into the underlying causes of the issues within their social world. The research process becomes a means of moving their voice from the margins into a place of centrality. In participatory case research, the case participants become contributing researchers.

\section{Conceptual Overview and Discussion}

Participatory research is research that is fully collaborative and emphasizes deliberate participation, contribution, empowerment and emancipation of all relevant parties in actively examining some issue, which participants experience as problematic. An outcome of the research process is to change and improve the social situation under investigation. Participatory case research is designed to address specific issues identified by local people, and the results are directly applied to the problems at hand. Therefore, it is ideally by the local people and for the local people. It has emerged in recent years as a significant methodology for intervention, development, and change within communities and groups. It is now promoted and implemented by many international development agencies. 
Guiding principles underlying participatory case research are:

- Participatory research is fundamentally conceptualized as a social action, change-oriented methodology. It promotes a dialogue between the local "insider" understandings of participants in a social context who are dissatisfied with the status quo and want change, and the general "outsider" understandings of social scientists and researchers. Meaningful participation insures that all partners are invested in the project results.

- There is an assumption of co-equal status of practitioner knowledge and expert knowledge.

- The formal boundaries between the traditional roles (researcher-subject/participant, knowledge producer-knowledge consumer, etc.) are reduced or eliminated in favor of a variety of interchangeable egalitarian roles. The research process is designed to enhance the knowledge and skills of all participants.

- The goal of the research process is to produce authentic knowledge that will directly benefit the participants and the program and/or community that is the focus of the case.

- The methods and techniques used are sensitive to the co-researcher's (the participant/program/community) culture, history, emotional life, and language. Consistent and persistent attempts are made to make the research activity inclusive to those participating at every step of the process. Project findings include the voices and interpretations of all collaborators.

- When combined with the cyclical, iterative processes of action research, participatory case studies incorporate concurrent and reciprocal levels of inquiry, observation, dialogue, and reflection with the traditional processes of case study research. 
- All accounts and reports reflect the perceptions of all stakeholders and are written in clear, everyday language.

Values place a central and salient role in participatory research. Explicit values embedded in this methodology are:

- Knowledge production and its use are mutually owned and are democratic in nature.

- Research must benefit those who contributed to it. Therefore, knowledge is to be used ethically and fairly.

- Researchers hold a deep appreciation for the human capacity to reflect, learn, and change. Participatory case researchers assume a strengths-based approach in which participants are encouraged to realize their potential by recognizing, using, and building on their own strengths and existing resources to accomplish their goals.

- There is an abiding commitment to social justice and non-violent social change.

- Research partnerships and relationships are characterized by equality, dialogue, mutual respect, inclusivity, and collaboration.

- In a participatory framework, objectivity is not the gold standard; rather critical subjectivity and reflexivity are valued.

\section{Applications}

A participatory approach to case study research demands that a significant amount of time and energy be invested in creating research relationships. This includes time to explore individual and mutual perceptions, expectations, and biases around what is known and experienced with regards to the targeted issue. Collaborative drafting of the research hypotheses or questions is done, as well as clarifications of the overall objectives of the project. Ethical issues that may emerge from the research process are explicitly discussed, and any issues related to formal ethics 
clearance (a tribal council or community ethics review board or an IRB) are talked about and resolved.

Decisions are then made collectively about the case study protocol: methodology; delineation and operationalization of the variables under investigation (if appropriate to the methodology); selection of the case study sites or profile of the participants to be recruited; sources of data; procedures for data collection; creation of the data collection instruments (e.g. interview protocol or questionnaire); logistics regarding implementation of the research project (e. g. site visits and data management procedures); and projected modes of data analysis. At each and every step, the research collaborators need to assess the skill and knowledge levels of the partners and provide scaffolded learning opportunities to gain whatever is lacking. As well, there may be times of considerable differences in approach or perspective, and this must be negotiated to everyone's satisfaction.

Collaboration during the analysis and dissemination phases is also crucial for the successful use of this participative method of case research. Meetings to outline the process of analysis and the procedures, or to generate code categories, codebooks, and code relationships (if using a qualitative methodology) not only allow the research partners to render these processes explicit, but also create opportunities for more authentic interpretations.

If a research report is generated, this is done in such a way as to represent the perspectives of all the research partners, and to be accessible to the community in general. Frequently, this takes the form of a presentation of the results or a performance as an alternate method of data representation that is open to the public.

\section{Critical Summary}

There are a number of challenges to carrying out participatory case research. It can be difficult to 
communicate with many participant/partners on an ongoing basis. However, this can be accomplished through newsletters, conference calls, frequent in-person meetings, and site visits. Since a fair amount of participatory case research is done with community groups, it can be a challenge to maintain the momentum of the project if there is a turnover in staff or a change in community leadership. Gaining a commitment to stick with the project is desirable, but not always feasible. When using a participatory case research methodology with multiple sites, it may be difficult to balance cross-site consistency with addressing questions that are of local interest and most salient to the participants. This can be accomplished by having a core set of questions and/or procedures, but making space for local questions that may draw on the same or different methodologies. The most challenging dimension is the need to invest time in all phases of the research process and in the cultivation of relationships. In order to be a good listener and partner, researchers require emotional intelligence, a democratic personality, and the skills to build truly collaborative partnerships.

There are, however, substantial benefits to this approach. Researchers can gain greater insights into issues or programs and their community contexts by having an equal partner who provides the "insider" view and knowledge. Researchers report that more effective questions and procedures are developed since they are framed in the language and perspective of the people who live in the context. As well, there is a high level commitment from the participants and the community to participate and this translates into higher response rates. Researchers can have greater confidence in the interpretation of the data, since it is grounded in the participant/partner's authentic experiences. And, last but not least, new relationships are formed, which contribute towards the betterment of individual and community life.

Participant benefits include: an increased sense of personal and community agency; an improved 
quality of life as changes in their social context take effect; positive development of knowledge and skills; leadership and relationship development; a sense of the whole and of community rather than isolation; and positive identity formation at an individual and collective level.

See also: Action Research; Power/Knowledge; Researcher-Participant Relationship

\section{Further reading and references:}

Freire, P. (1982). Creating alternative research methods. Learning to do it by doing it. In B. Hall, A. Gillette \& R. Tandon (Eds.), Creating knowledge: A monopoly (pp. 29-37). New Delhi: Society for Participatory Research in Asia.

Guba, E., \& Lincoln, Y. (2005). Paradigmatic controversies, contradictions, and emerging confluences. In N. Denzin \& Y. Lincoln (Eds.), N. Denzin \& Y. Lincoln (Eds.), The Sage handbook of qualitative research ( $3^{\text {rd }}$ ed.). (pp. 191-215). Thousand Oaks, CA: Sage.

Maguire, P. (1987). Doing participatory research: A feminist approach. Amherst, MA: Center for International Education.

Merriam, S., \& Simpson, E. (1984). A guide to research for educators and trainers of adults. Malabar, FL: Robert E. Krieger Publishing. 